\title{
Low-intensity pulsed ultrasound alleviating myelosuppression of Sprague-Dawley rats after combined treating by paclitaxel and carboplatin
}

\author{
Dong Luo, Wenzhi Chen, Wei Wang, Junlin Chen, Haopeng Xu, Jinyun Chen, Yan Wang \\ State Key Laboratory of Ultrasound in Medicine and Engineering, College of Biomedical Engineering, Chongqing Key Laboratory of Biomedical \\ Engineering, Chongqing Medical University, Chongqing, China \\ Contributions: (I) Conception and design: Y Wang; (II) Administrative support: W Chen; (III) Provision of study materials or patients: J Chen; (IV) \\ Collection and assembly of data: D Luo, W Wei, J Chen; (V) Data analysis and interpretation: D Luo, H Xu; (VI) Manuscript writing: All authors; (VII) \\ Final approval of manuscript: All authors. \\ Correspondence to: Yan Wang. College of Biomedical Engineering, Chongqing Medical University, Chongqing 400016, China. \\ Email: wangyancq@cqmu.edu.cn.
}

\begin{abstract}
Background: Our team has previously reported that low intensity pulsed ultrasound (LIPUS) can alleviate myelosuppression in rats induced by single chemotherapy drugs. But in clinics, chemotherapy is often performed with multiple drugs simultaneously. To be closer to the clinical status quo, this experiment was designed to show whether it was the same effect of LIPUS on myelosuppression caused by combination therapy of chemotherapy drugs.
\end{abstract}

Methods: The rat model of myelosuppression was established by continuous injection of paclitaxel and carboplatin for 4 days. These myelosuppressive rats were randomly divided into LIPUS group ( $n=40)$ and control group $(\mathrm{n}=40)$. The LIPUS group was given continuous LIPUS irradiation for 7 days, while the control group was given sham irradiation (no energy output). The evaluation of blood cells counts, Hematoxylin-Eosin staining (H\&E staining), scanning electron microscopy, enzyme-linked immunosorbent assay (ELISA) and real-time quantitative PCR (qPCR) was then performed.

Results: The results showed in the LIPUS group the blood cells count, hematopoietic tissue of bone marrow, the colonies formed from adhering of bone marrow stromal cells, levels of hematopoietic regulators and adhesion molecules all increased (LIPUS group vs. control group, $\mathrm{P}<0.05$ ).

Conclusions: The results indicated that LIPUS can relieve myelosuppression induced by combined treatment of paclitaxel and carboplatin. The mechanisms may be LIPUS can increase the levels of hematopoietic regulators and adhesion molecules.

Keywords: Myelosuppression; low intensity pulsed ultrasound (LIPUS); hematopoiesis; paclitaxel; carboplatin

Submitted Oct 13, 2020. Accepted for publication Feb 07, 2021.

doi: $10.21037 /$ tcr-20-3035

View this article at: http://dx.doi.org/10.21037/tcr-20-3035

\section{Introduction}

Most of the chemotherapeutic drugs will kill cancer cells and normal tissue cells simultaneously, causing a variety of toxic side effects such as digestive tract reaction, central nervous system reaction, cardiovascular disease and myelosuppression (1-5). Among these toxic and side effects, hematopoietic damage triggered off by chemotherapeutic drugs is particularly serious. The hematopoietic tissue and cell will be damaged mostly then lead to the number of peripheral blood cells released from bone marrow decreasing, especially the leukocytes decline seriously in a short time. There are symptoms such as anemia, hemorrhage, infection, even life-threatening (6) because of the myelosuppression. Re-establishing hematopoiesis 
and immune system are the key to treating these diseases. At present, the treatments rely mainly on leukocytes drugs (7), platelet transfusion (8) and haemopoietic growth factors (9) therapy to improve the basic conditions of patients. But these methods have disadvantages for some patients such as transfusion reactions and transfusiontransmitted infections (10), expensive cost $(11,12)$, and so on. Therefore, a safer, more effective and economical method should be found to promote medullary hematopoiesis function to support cancerous people complete the course of chemotherapy successfully and improve their quality of life.

As an emerging physical therapy, low-intensity pulsed ultrasound (LIPUS) has been approved as a safe noninvasive method for the treatment of bone healing, softtissue regeneration, inhibition of inflammation, noninvasive neuromodulation, and other applications (13). Our previous experimental study showed that LIPUS can promote the proliferation, differentiation and migration of bone marrow mesenchymal stem cells (14-16), and also found that LIPUS can treat leukopenia caused by cyclophosphamide chemotherapy and elevate leukocytes count after cytarabine chemotherapy $(17,18)$.

But, the above results of our research only use single drugs to induce myelosuppression. In consideration of current clinical application, different cell cycle chemotherapy drugs are often used to treat cancer in combination, such as paclitaxel and carboplatin $(19,20)$. We thought animal models should be conducted in strict simulation of clinical diagnosis and treatment to disease in order to be closer to the clinical status quo. So, in this research we triggered the hematopoietic damages of SD rats by paclitaxel plus carboplatin. This research was further explored whether LIPUS can reconstruct the hematopoietic function in myelosuppression induced by paclitaxel plus carboplatin combination chemotherapy. The mechanism of treating effects of LIPUS on hematopoietic damage will be tried to explain. The results might introduce a new method to treat hematopoietic damage caused by combined chemotherapy in clinics in the future. We present the following article in accordance with the ARRIVE reporting checklist (available at: http://dx.doi.org/10.21037/tcr-20-3035).

\section{Methods}

\section{Animal experiments}

A total of 80 healthy Sprague-Dawley rats (SD rats, male and female, SPF grade, weight $175 \pm 25 \mathrm{~g}$ ) were purchased from the Animal Experimental Center of Chongqing Medical University. The feeding environment of all rats was uniformly standardized (temperature: $23 \pm 0.5{ }^{\circ} \mathrm{C}$, humidity: $45 \% \pm 5 \%, 12 \mathrm{~h}$ light and dark cycle). This project was according to the ethical standards of the Experimental Animal Ethics Committee of Chongqing Medical University. Experiments were performed under a project license (SCXK 2018-0003) granted by ethics board of Chongqing Medical University, in compliance with all national and institutional guidelines for the care and use of animals.

In order to establish a model of myelosuppression, all rats were injected intraperitoneally according to the dose of paclitaxel $(10 \mathrm{mg} / \mathrm{kg}$, Aladdin Reagent Co., Ltd, Shanghai, China) plus carboplatin $(16 \mathrm{mg} / \mathrm{kg}$, Aladdin Reagent Co., Ltd, Shanghai, China) dissolved with physiological saline for 4 consecutive days. The rats were randomly divided into two groups: control group (injecting drugs + no energy output from LIPUS device, $\mathrm{n}=40$ ) and LIPUS group (injecting drugs + LIPUS, $\mathrm{n}=40$ ).

After drug administration for 4 days, the LIPUS group began treatment with LIPUS device (Chongqing Haifu Medical Technology Co., Ltd., China). The parameters of LIPUS were involved as follows: spatial-average temporalaverage intensity $\left(\mathrm{I}_{\mathrm{SATA}}\right) 0.2 \mathrm{~W} / \mathrm{cm}^{2}$, frequency $0.27 \mathrm{MHz}$, duty cycle $20 \%$ and pulse repetition frequency $1 \mathrm{KHz}$. The position of treating was the metaphyseal of the right hind limb femur. Before LIPUS treatment, the surface of the right hind limb skin was depilated, disinfected, and covered with the ultrasonic coupling agent. Then, LIPUS started to work. The control group was handled as the same as the LIPUS group except for no energy output from LIPUS device. The treatment time was $20 \mathrm{~min} /$ day, for 7 consecutive days.

The mental state of the rats, diarrhea, skin and hair color, exercise, weight changes and time of death were observed and recorded during the experiment.

\section{Detection of blood cell counts}

To detect the counts of blood cell, the rats ( $\mathrm{n}=6$, per group and per time point) were chosen randomly on the days $0,4,7,9,11,14$ and 18 . The $0.5 \mathrm{~mL}$ heart blood was extracted from the rats anesthetized and quickly injected into the disposable blood collection tube (including EDTA, Yulin medical equipment Co., Ltd., China). The blood in the blood collection tube was mixed upside down 
Table 1 Primer sequences

\begin{tabular}{lll}
\hline Gene & Upstream primer (5'-3') & Downstream primer (5'-3') \\
\hline SCF & GCGCTGCCTTTCCTTATGAA & GGAAGATTCGCCACCAGTTT \\
ICAM-1 & CGCCAGAGGAAGATCAGGAT & AGGTGGGTGAGGGGTAAATG \\
VCAM-1 & GCCTCGCTAAGTTACACAGC & AGGTCAGGTTCACAGGACTG \\
$\beta$-actin & AGTGCTGTGGGTGTAGGTAC & GCAAAGAGGGCAAGAACACA \\
\hline
\end{tabular}

SCF, stem cell factor; ICAM-1, intercellular adhesion molecule-1; VCAM-1, vascular cell adhesion molecule-1.

for anticoagulation, and was detected by automatic fiveclassification blood analyzer (XT-2000i, SYSMEX, Japan) within $2 \mathrm{~h}$.

\section{Real-time quantitative PCR}

To examine the expression of stem cell factor (SCF), intercellular adhesion molecule-1 (ICAM-1) and vascular cell adhesion molecule-1 (VCAM-1), the total RNA was extracted on the 11th day and purified from bone marrow using Trizol reagent (Invitrogen, USA) and reverse transcribed into cDNA according to the instructions of procedures (Shanghai Westang BIO-TECH Co., Ltd., Shanghai, China). Next, the PCR reaction was quantified in real time using SYBR Green (Invitrogen, USA). After the reaction, the fluorescence signal data was analyzed by BioRad Prime PCR software. The primers were designed by Primer express 3.0 software (ABI, USA) and synthesized by Shanghai Shenggong Bioengineering Co., Ltd. (Table 1).

\section{Hematoxylin-Eosin staining}

To investigate the change of bone marrow, the rats $(n=6)$ were euthanized on the days 0,4 , and 11 , the right hind limb hair was shaved and the skin was disinfected. Then the right femur was dissected and the bone marrow was fixed in 4\% paraformaldehyde (Chongqing Boer Biotechnology Co., Ltd., Chongqing, China). After dehydration, decalcification, embedding, sectioning and staining, the morphology of hematopoietic tissue was surveyed under optical microscope (BX51, Olympus, Germany) with the same magnification and each tissue specimen was observed with the same 10 visual fields. Bone marrow tissue sections (10 images per sample) were quantitatively analyzed by Image J (IBM, Armonk, NY, USA) software, and the proportion of hematopoietic tissue in the above samples was calculated.

\section{Scanning electron microscope}

To observe the change of hemopoietic tissue, on the days 0,4 , and 11 the part of the femur was taken $1 \mathrm{~cm}$ long and then cut along a major axis. After being fixed in $2.5 \%$ glutaraldehyde fixative (Chongqing Boer Biotechnology Co, Ltd, Chongqing, China) at $4{ }^{\circ} \mathrm{C}$ over $4 \mathrm{~h}$, the samples were handled by dehydration, drying, coating, at last detected under a scanning electron microscope (Hitachi, Tokyo, Japan) (21).

\section{Enzyme-linked immunosorbent assay (ELISA)}

To detect the level of Human granulocyte-macrophage colony stimulating factor (GM-CSF) and interleukin 3 (IL-3), the femur was cut on days 0,4 , and 11 , then the supernatant was isolated from the bone marrow in the femur by centrifugation at $1,000 \mathrm{r} / \mathrm{min}$ for $10 \mathrm{~min}$. According to the instructions of the ELISA kit (Shanghai Westang BIOTECH Co., Ltd., Shanghai, China), the GM-CSF and IL-3 in bone marrow supernatant were detected.

\section{Statistical analysis}

The data was analyzed with statistical software SPSS 25.0 (IBM SPSS, Chicago, IL, USA). All data was expressed as mean \pm standard deviations, one-way analysis of variance was used to compare multi-sample means, and LSD-t method was used to compare the two pairs. $\mathrm{P}<0.05$ was considered to be statistically significant.

\section{Results}

\section{The counts of peripheral blood cells}

The counts of white blood cells and platelets decreased after paclitaxel and carboplatin administration for 4 days. Compared with the control group, the counts of white 

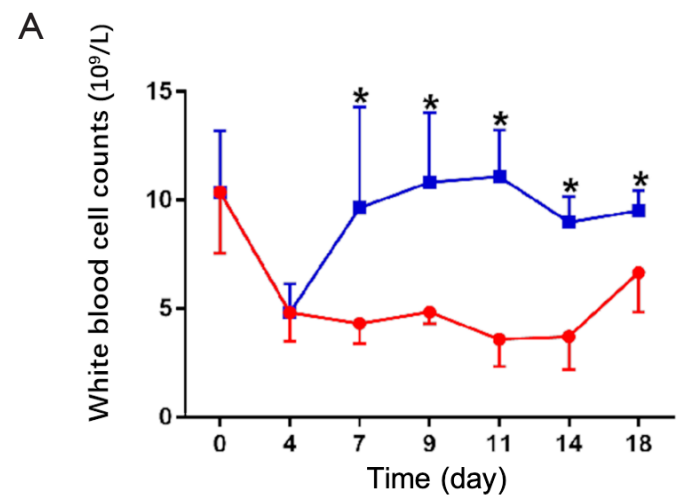

C

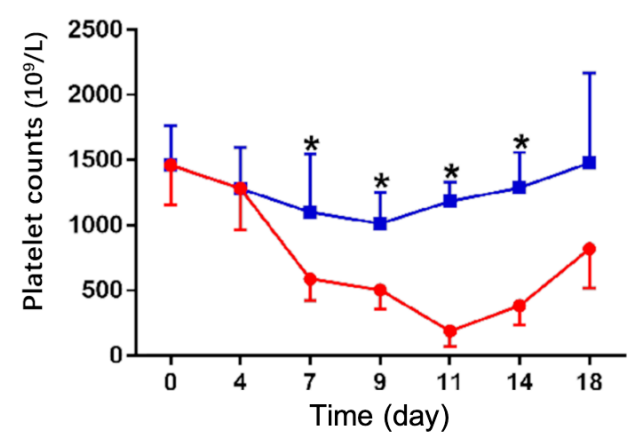

B

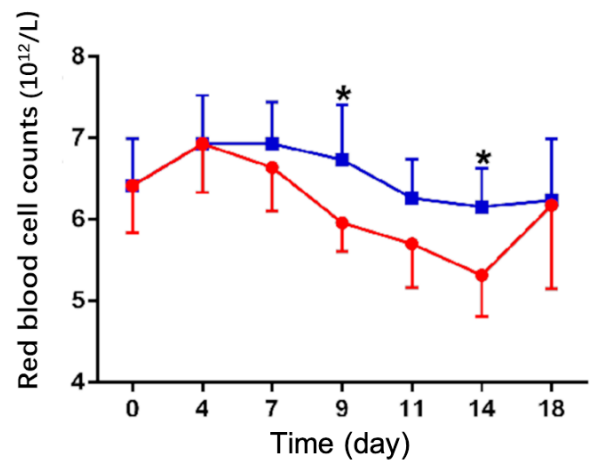

$\rightarrow$ Control Group

- LIPUS Group

Figure 1 The counts changes of blood cells in LIPUS group and control group. ${ }^{*}, \mathrm{P}<0.05$.

blood cells in LIPUS group increased significantly on the 7th, 9th, 11th, 14th, and 18th day $(\mathrm{P}<0.05$, Figure $1 A)$, and which in the control group stayed at a low level. The counts of red blood cells in the LIPUS group were higher than control group significantly on 9th and 14 th day $(\mathrm{P}<0.05$, Figure $1 B$ ). The counts of platelets in the LIPUS group increased significantly on 7 th, 9th, 11th, and 14th day $(\mathrm{P}<0.05$, Figure $1 C)$. The results suggested that LIPUS can alleviate the decline of blood cells major from the 7 th to 14th day after modeling.

\section{The expressions of SCF, ICAM-1 and ACVM-1}

The mRNA levels of SCF, ICAM-1, and VCAM-1 were compared. On the 11th day after modeling, the level of SCF in LIPUS group and control group was significantly higher than that on the 4th day respectively $(\mathrm{P}<0.05)$. However, on the 11th day, there was no significant difference in SCF between the LIPUS group and control group $(\mathrm{P}>0.05$, Figure 2). The ICAM-1 in the LIPUS group and control group were significantly higher than those after modeling $(\mathrm{P}<0.05)$. There was significantly different between the LIPUS group and the control group $(\mathrm{P}<0.05$, Figure 2$)$. The
VCAM-1 in the LIPUS group and the control group were significantly higher than those after modeling $(\mathrm{P}<0.05)$. The LIPUS group was significantly different from the control group $(\mathrm{P}<0.05$, Figure 2). The results suggested that LIPUS can promote the secretion of VCAM-1 and ICAM-1, but the effect on SCF is not obvious.

\section{Pathological changes in bone marrow}

The normal bone marrow hematopoietic tissue of rat was rich and the structure is complete (Figure $3 A$ ). After drug administration, myeloproliferative declined, megakaryocytes decreased or disappeared, fat cells count increased (Figure 3B). On the 11th day, the hematopoietic tissue of the control group increased, but significant suppression was still observed (Figure 3C). The bone marrow failure in LIPUS group was improved, hematopoietic tissue was observed increased and returned to normal levels compared to the control group (Figure 3D). The results suggested that bone marrow failure in the control group was in suppression state for a long time, but the in the LIPUS group showed recovery obviously.

Quantitative analysis for the bone marrow histological 
SCF

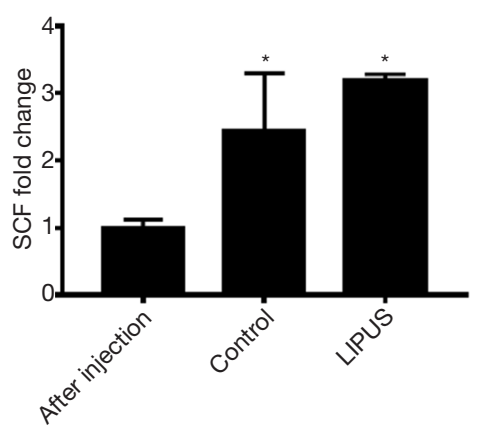

ICAM1

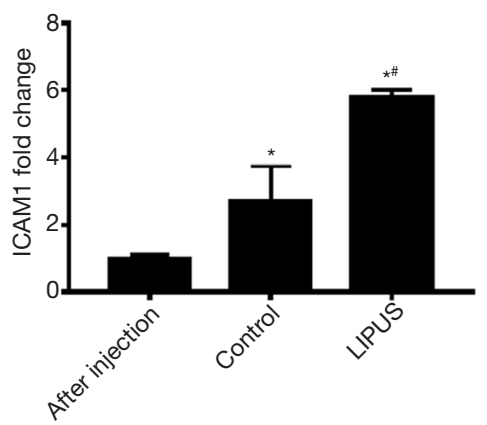

VCAM1

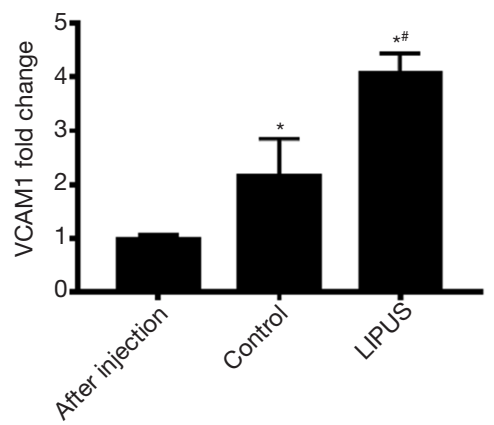

Figure 2 Hematopoietic-related factors [stem cell factor (SCF), intercellular adhesion molecule-1 (ICAM-1) and vascular cell adhesion molecule-1 (VCAM-1)] in rats' bone marrow on the 11 th day. * $\mathrm{P}<0.05$ LIPUS group and control group versus those in rats after drug injection and before LIPUS treatment (on the 4th day). ", $\mathrm{P}<0.05$ LIPUS group versus control group.
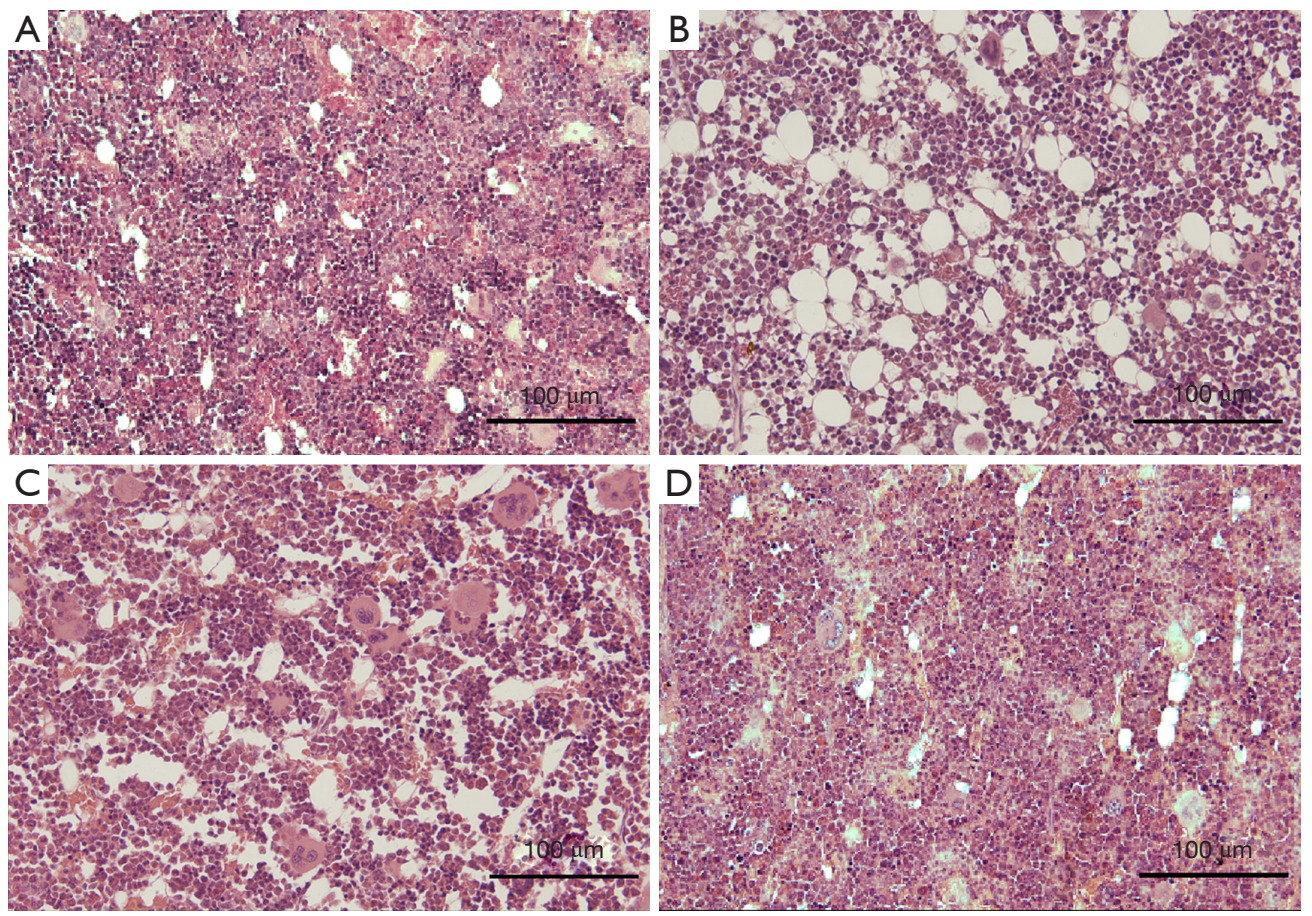

Figure 3 Sections of bone marrow in rats (Hematoxylin-Eosin staining, original magnification: $\times 400$ ). (A) Normal bone marrow tissue. (B) The bone marrow tissue in LIPUS group on the 11th day. (C) After drug injection (on the 4th day). (D) The bone marrow tissue in the control group on the 11 th day.

sections were performed (Figure 4). The areas of hematopoietic tissue decreased on the 4th day of the control group $(\mathrm{P}<0.05)$ and the day 0 and 11 th days were also statistically significant $(\mathrm{P}<0.05)$. There was no difference between the 4th day and the 11th day $(\mathrm{P}>0.05)$. It indicated that there was still suppressed significantly in hematopoietic tissue until the 11 th day (Figure $4 A$ ). On the 4th day, hematopoietic tissues in the LIPUS group decreased significantly $(\mathrm{P}<0.05)$. There was a significant difference between the 11th day and the 4th day $(\mathrm{P}<0.05)$. There was no difference between the day 0 and 11 th days. It suggested that the hematopoietic tissue recovered on the 11th day (Figure $4 B$ ). There was a significant difference between the LIPUS group and control group on the 11th day $(\mathrm{P}<0.05)$. 
A

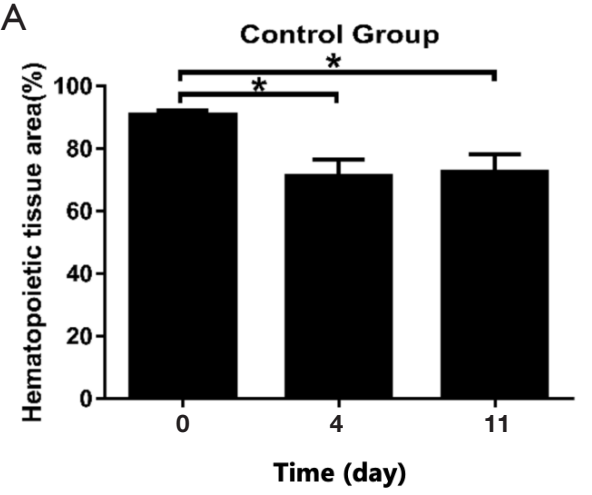

C

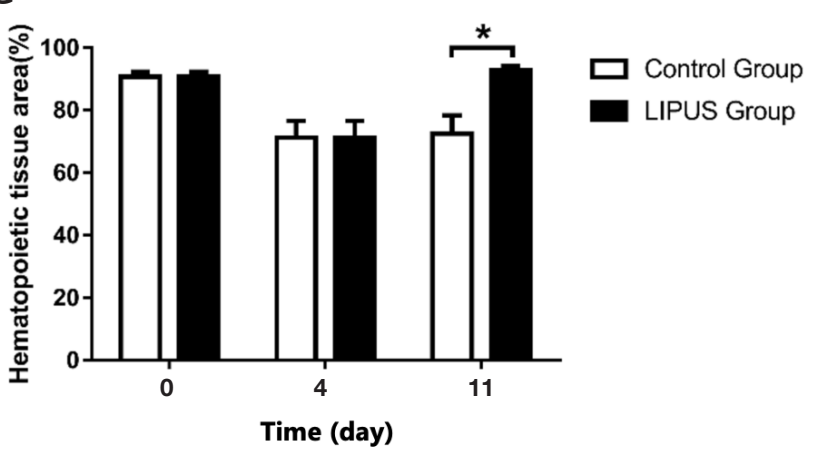

B

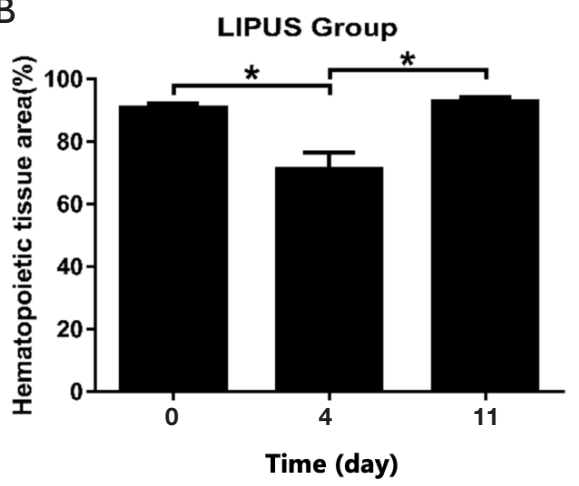

Time (day)

Figure 4 The area of hematopoietic tissue in rats. (A) The comparation in control group on the day 0 , 4th and 11 th day. (B) The comparation in LIPUS group on the day 0 , 4th and 11th day. (C) The comparison between LIPUS group and control group on the day 0 , 4th and 11th day. *, $\mathrm{P}<0.05$.

The results showed that hematopoietic tissue was increased under the LIPUS treatment (Figure 4C).

\section{Bone marrow structure}

The results were analyzed by H\&E staining and scanning electron microscopy. In normal rats, bone marrow tissue was gathered to form a colony (Figure 5A). However, after drug administration, the adherent cells are significantly reduced and less gathered (Figure 5B). On the 11th day, the cells began to re-form new small colonies, but the number of attached cells did not increase (Figure 5C). In contrast, on the 11th day, cells in the LIPUS group were abundant and large colonies were formed (Figure 5D). It showed that LIPUS promoted the adhesion of bone marrow cells and helped the bone marrow cells to form colonies.

\section{Levels of GM-CSF and IL-3}

The expression of GM-CSF and IL-3 in rat bone marrow supernatants was compared on the day 0,4 th and 11 th day.
The level of GM-CSF in the control group rose on the 4th day first, and then showed a significant decrease on the 11th day. However, although the LIPUS group decreased on the 11th day, the level of GM-CSF was still much higher than in the control group (Figure $6 A, B, C, \mathrm{P}<0.05$ ). The level of IL-3 in LIPUS group began to decline on the 4th day, and was significantly higher than in the control group on the 11th day (Figure $6 D, E, F, \mathrm{P}<0.05$ ).

\section{Discussion}

In recent years, chemotherapy is still the main treatment for malignant tumors in the middle and advanced stages. The chemotherapy is often applied with cell cycle-specific drugs combined with cell cycle non-specific drugs in clinic currently. The utilizing of combination chemotherapy drugs has been shown higher than the single drug because of the better therapeutic effects and fewer side effects. However, the current animal model of hematopoietic damage has been induced still mainly by single drugs, which are not inconsistent with the clinical treatment plan. The periodic 

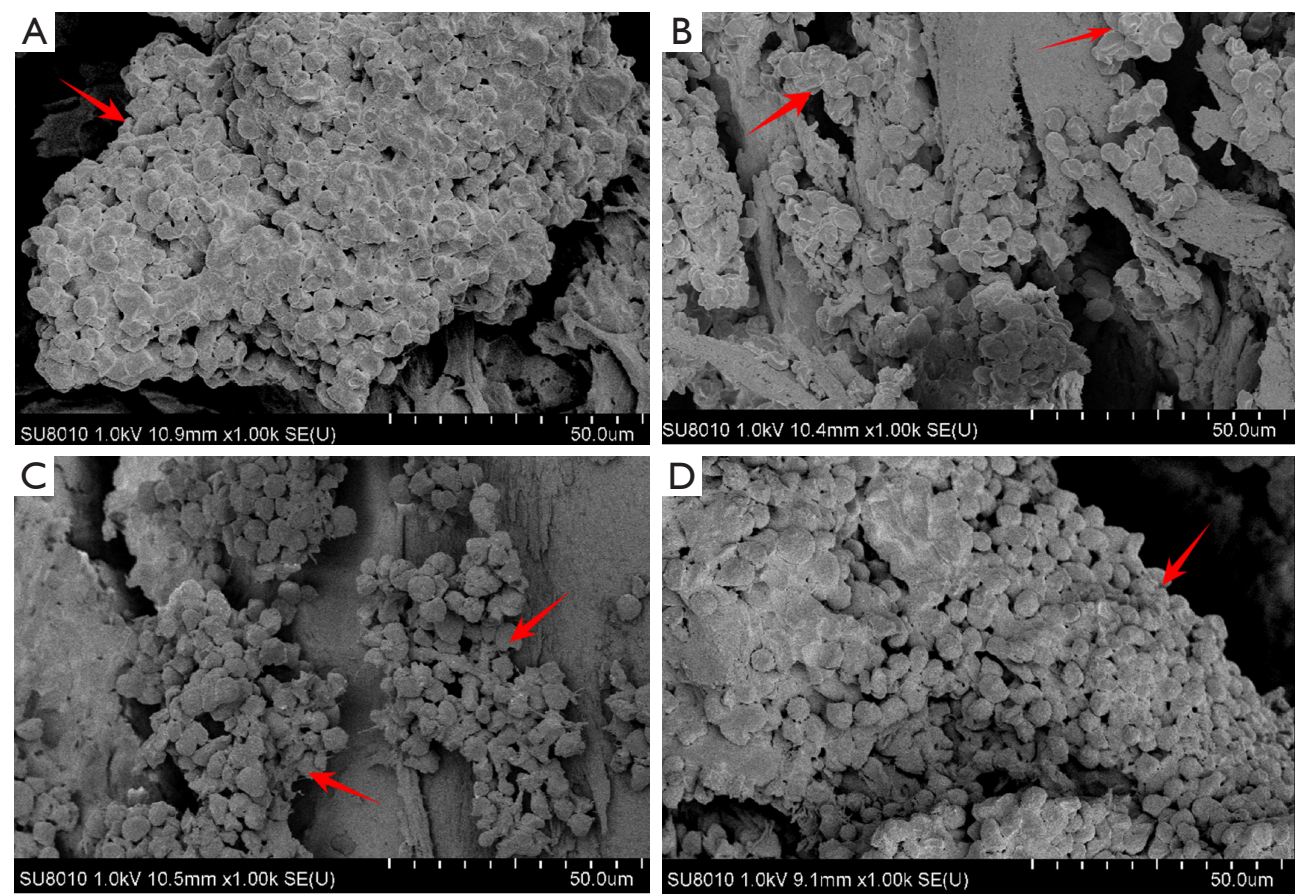

Figure 5 Scanning electron microscope image of rat bone marrow (original magnification: $\times 1 \mathrm{k}$ ). (A) Normal bone marrow tissue. (B) The bone marrow tissue on the 4th day. (C) The bone marrow tissue on the 11th day in control group. (D) The bone marrow tissue on the 11 th day in LIPUS group. The red arrows indicate bone marrow cell aggregates.

specific drug paclitaxel plus the cell cycle non-specific drug carboplatin is widely used in clinical treatment, especially in the treatment of non-small cell lung cancer (22). So, the animal hematopoietic model was tried to and achieved to make by paclitaxel plus carboplatin in this research to be fitting in with the clinical requirements.

The paclitaxel plus carboplatin also causes bone marrow failure when killing tumor cells. The main damages of the two drugs are to make leukocytes and platelet counts be decreased, which will lead to the body's immune decline, increased susceptibility, bleeding tendency and many other side effects, and may even be life-threatening. In this experiment, the leukocyte and platelets counts declined after paclitaxel and carboplatin administration for 4 days, which was in keeping with those side effects talked above. The results demonstrated that it is feasible to construct a bone marrow suppressive model with combined chemotherapy drugs.

Currently, with the rapid and comprehensive development of non-invasive method, LIPUS has been widely used in articular cartilage regeneration (23), soft tissue repair (24), anti-infection and infarction (25), nerve regeneration (26) and vascular thrombolysis (27), myelosuppression researched by our past studies $(17,18,21)$, and so on. LIPUS is deserved as a new method with the advantages of effectiveness, safety and economy. So, in this investigation, the treatment effects of LIPUS on bone marrow failure rats induced by paclitaxel plus carboplatin were observed.

The results of this study showed that LIPUS had the effect on making the hematopoietic damaged recovered. Through blood analysis, LIPUS was proven to promote the increase of leukocytes and platelets after paclitaxel combined with carboplatin chemotherapy. These results were the same as those which had been reported by our team $(17,18,21)$. But the effect on erythrocytes was not as obvious as on leukocytes and platelets. The decline of erythrocytes was slowed down merely by LIPUS. The reason maybe the normal erythrocytes lifespan is about 120 days, which is difficult to change in the short term.

It was reported that the SCF is the key to survival, proliferation and differentiation of early hematopoietic stem cells (28). The ICAM-1 and VCAM-1 are two adhesion molecules expressed by bone marrow stromal cells. The VCAM-1 was involved in the homing of early blood progenitor cells (29) and ICAM-1 could activate specific kinase through phosphorylation, leading to increased 
A

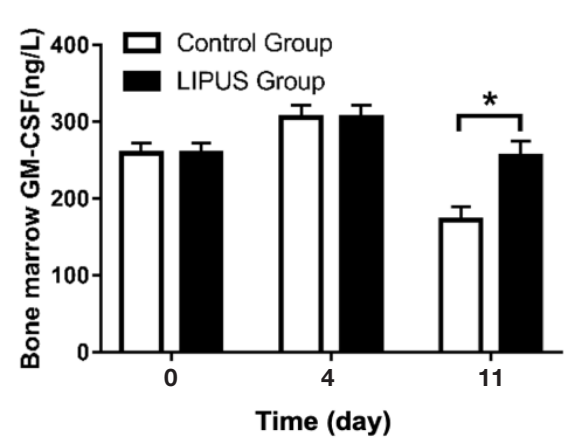

D

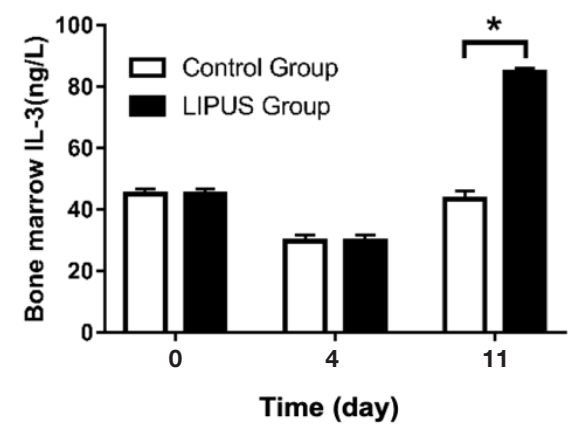

B

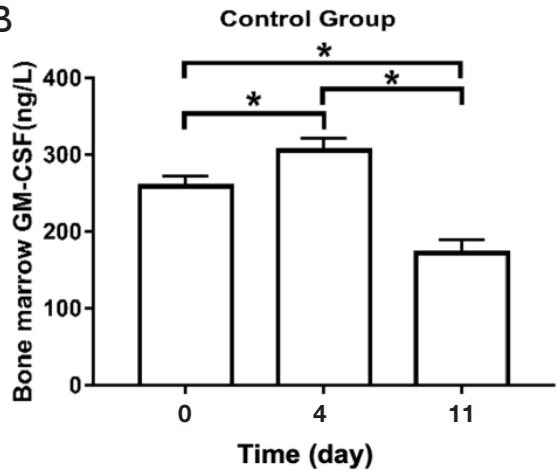

$\mathrm{E}$

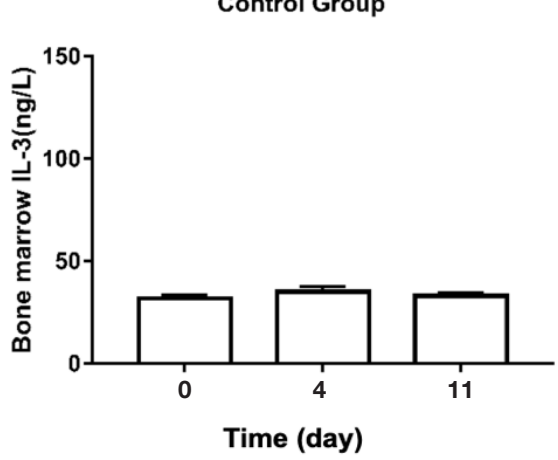

C

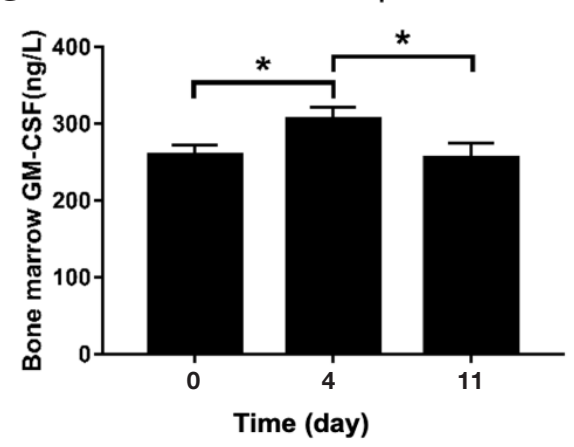

$\mathrm{F}$

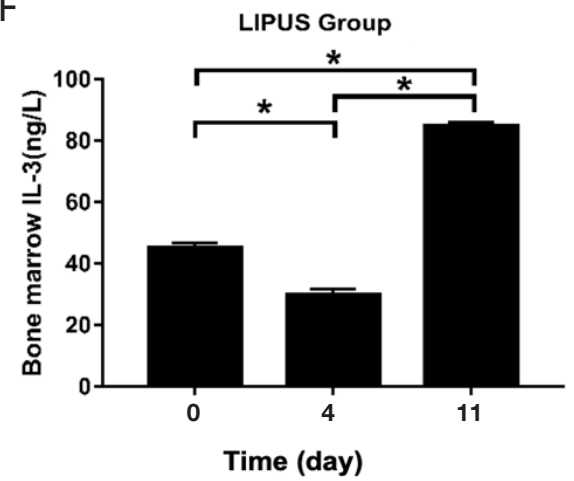

Figure 6 The levels of Human granulocyte-macrophage colony stimulating factor (GM-CSF) and interleukin 3 (IL-3) in the bone marrow were detected by enzyme-linked immunosorbent assay (ELISA) on the day 0, 4th, and 11th day. (A-C) The levels of GM-CSF comparation between LIPUS group and control group, in control group and in LIPUS group on the day 0, 4th and 11th day; (D-F) The levels of IL-3 comparison between LIPUS group and control group, in control group and in LIPUS group on the day 0 , 4th and 11 th day. ${ }^{*}, \mathrm{P}<0.05$.

transcription factor activation and cytokine production, increased expression of cell membrane proteins, and reactive oxygen species (30). The SCF detection result indicated that LIPUS had no significant effect on the secretion of SCF. Zhao et al. (31) had found that SCF promotes hematopoiesis and angiogenesis is a slow process, and the need for SCF is elevated. For a period of time, it would take more time to increase the SCF than ICAM-1 and VCAM-1. Through the detection of ICAM-1 and VCAM-1, it was found that there was a significant increase in LIPUS group and control group in 11th days after drugs administration. VCAM1 levels have been significantly improved. Our results suggested that LIPUS activated adhesion and proliferation of bone marrow cells and the like possibly by raising the level of VCAM-1 and ICAM-1, thereby improving the hematopoietic damage.

Compared with the control group, the increase in hematopoietic tissue was significant in the LIPUS group. After LIPUS treatment, hematopoietic tissue had recovered reaching the normal, which indicated that LIPUS can rapidly promote hematopoietic tissue recovery. Carbonneau et al. (32) found that the colony forming ability of bone marrow stromal cells and osteoblasts significantly decreased after chemoradiotherapy even caused hematopoietic injury. In the experiment, it can be seen that large colonies adhere to the trabecular bone after LIPUS treatment. The results indicated that LIPUS promoted the adhesion of bone marrow stromal cells to form colonies, thus repairing the hematopoietic damage after combined chemotherapy.

Both GM-CSF and IL-3 are hematopoietic regulators (33). GM-CSF mainly stimulates the cloning of granulocytes and macrophages $(\mathrm{G} / \mathrm{M})$ and enhances the function of mature cells (34). The main role of IL-3 is to stimulate G/M colonies (35). In the research, LIPUS promoted the secretion of GM-CSF and IL-3, thereby accelerating cell maturation and adhesion. Finally, the hematopoietic damage was also improved.

Of course, further research is needed to clearly understand the specific effects and mechanisms of LIPUS on the immune system. 
In summary, long-term stable bone marrow failure can be induced by the cell cycle-specific drug paclitaxel plus cell cycle non-specific drug carboplatin combination chemotherapy, which was consistent with clinical manifestations of the two drugs. The hematopoietic damage can be improved by LIPUS, which mechanisms maybe through correcting blood cell decline, increasing hematopoietic tissue, enhancing the levels of ICAM-1 and VCAM-1, and promoting the secretion of GM-CSF and IL-3.

\section{Acknowledgments}

Funding: None.

\section{Footnote}

Reporting Checklist: The authors have completed the ARRIVE reporting checklist. Available at http://dx.doi. org/10.21037/tcr-20-3035

Data Sharing Statement: Available at http://dx.doi. org/10.21037/tcr-20-3035

Peer Review File: Available at http://dx.doi.org/10.21037/tcr20-3035

Conflicts of Interest: All authors have completed the ICMJE uniform disclosure form (available at http://dx.doi. org/10.21037/tcr-20-3035). The authors have no conflicts of interest to declare.

Ethical Statement: The authors are accountable for all aspects of the work in ensuring that questions related to the accuracy or integrity of any part of the work are appropriately investigated and resolved. Experiments were performed under a project license (SCXK 2018-0003) granted by ethics board of Chongqing Medical University, in compliance with all national and institutional guidelines for the care and use of animals.

Open Access Statement: This is an Open Access article distributed in accordance with the Creative Commons Attribution-NonCommercial-NoDerivs 4.0 International License (CC BY-NC-ND 4.0), which permits the noncommercial replication and distribution of the article with the strict proviso that no changes or edits are made and the original work is properly cited (including links to both the formal publication through the relevant DOI and the license). See: https://creativecommons.org/licenses/by-nc-nd/4.0/.

\section{References}

1. Javarappa KK, Tsallos D, Heckman CA. A multiplexed screening assay to evaluate chemotherapy-induced myelosuppression using healthy peripheral blood and bone marrow. SLAS Discov 2018;23:687-96.

2. Staff NP, Grisold A, Grisold W, et al. Chemotherapyinduced peripheral neuropathy: a current review. Ann Neurol 2017;81:772-81.

3. Yoshimura A, Noro R, Miyanaga A, et al. The east Japan chesters group, combination chemotherapy of alternating etoposide and carboplatin with weekly administration of irinotecan and cisplatin in extensive-stage small-cell lung cancer. Anticancer Res 2012;32:4473-8.

4. Varricchi G, Marone G, Mercurio V, et al. Immune checkpoint inhibitors and cardiac toxicity: an emerging issue. Curr Med Chem 2018;25:1327-39.

5. Glass CK, Mitchell RN. Winning the battle, but losing the war: mechanisms and morphology of cancer-therapyassociated cardiovascular toxicity. Cardiovasc Pathol 2017;30:55-63.

6. Barreto JN, McCullough KB, Ice LL, et al. Antineoplastic agents and the associated myelosuppressive effects: a review. J Pharm Pract 2014;27:440-6.

7. Lalami $Y$, Klastersky J. Impact of chemotherapy-induced neutropenia (CIN) and febrile neutropenia (FN) on cancer treatment outcomes: an overview about well-established and recently emerging clinical data. Crit Rev Oncol Hematol 2017;120:163-79.

8. Crighton GL, Estcourt LJ, Wood EM, et al. A therapeuticonly versus prophylactic platelet transfusion strategy for preventing bleeding in patients with haematological disorders after myelosuppressive chemotherapy or stem cell transplantation. Cochrane Database Syst Rev 2015;9:CD010981.

9. Ketley NJ, Newland AC. Haemopoietic growth factors. Postgrad Med J 1997;73:215-21.

10. Malouf R, Ashraf A, Hadjinicolaou AV, et al. Comparison of a therapeutic-only versus prophylactic platelet transfusion policy for people with congenital or acquired bone marrow failure disorders. Cochrane Database Syst Rev 2018;5:CD012342.

11. Chen W, Rizzieri D, Drago S. Safety of growth factor administration for leukapheresis in those with WBC counts greater than 60,000/microl. J Clin Apher 2015;30:28-31. 
12. Chen SH, Yang SH, Chu SC, et al. The role of donor characteristics and post-granulocyte colony-stimulating factor leukocytes counts in predicting the adverse events and yields of stem cell mobilization. Int J Hematol 2011;93:652-9.

13. Jiang X, Savchenko O, Li Y, et al. A review of low-intensity pulsed ultrasound for therapeutic applications. IEEE Trans Biomed Eng 2019;66:2704-18.

14. He R, Zhou W, Zhang Y, et al. Combination of lowintensity pulsed ultrasound and C3H10-T1/2 cells promotes bone-defect healing. Int Orthop 2015;39:2181-9.

15. He R, Chen J, Jiang J, et al. Synergies of accelerating differentiation of bone marrow mesenchymal stem cells induced by low intensity pulsed ultrasound, osteogenic and endothelial inductive agent. Artif Cells Nanomed Biotechnol 2019;47:674-84.

16. Chen J, Jiang J, Wang W, et al. Low intensity pulsed ultrasound promotes the migration of bone marrowderived mesenchymal stem cells via activating FAKERK1/2 signalling pathway. Artif Cells Nanomed Biotechnol 2019;47:3603-13.

17. Liu B, Luo Y, Luo D, et al. Treatment effect of low intensity pulsed ultrasound on leukopenia induced by cyclophosphamide in rabbits. Am J Transl Res 2017;9:3315-25.

18. Zhang Y, He RX, Zhou WC, et al. Influence of low intensity pulsed ultrasound on chemotherapy-induced leukopenia and thrombocytopenia by Ara-C in rabbit. Journal of Chongqing Medical University 2015;40:1503-9.

19. O'Brien MER, Sarker D, Bhosle J, et al. A phase I study to assess afatinib in combination with carboplatin or with carboplatin plus paclitaxel in patients with advanced solid tumors. Cancer Chemother Pharmacol 2018;82:757-66.

20. Chan JK, Brady MF, Penson RT, et al. Weekly vs. every-3week paclitaxel and carboplatin for ovarian cancer. N Engl J Med 2016;374:738-48.

21. Liu B, Chen W, Jiang J, et al. Treatment effect of low-Intensity pulsed ultrasound on benzene- and cyclophosphamide-induced aplastic anemia in rabbits. Phys Ther 2019;99:1443-52.

22. Langer CJ, Leighton JC, Comis RL, et al. Paclitaxel and carboplatin in combination in the treatment of advanced non-small-cell lung cancer: a phase II toxicity, response, and survival analysis. J Clin Oncol 1995;13:1860-70.

23. Jia XL, Chen WZ, Zhou K, et al. Effects of low-intensity pulsed ultrasound in repairing injured articular cartilage. Chin J Traumatol 2005;8:175-8.

24. Lu H, Liu F, Chen H, et al. The effect of low-intensity pulsed ultrasound on bone-tendon junction healing: Initiating after inflammation stage. J Orthop Res 2016;34:1697-706.

25. Yang PF, Li D, Zhang SM, et al. Efficacy of ultrasound in the treatment of osteoarthritis of the knee. Orthop Surg 2011;3:181-7.

26. Mourad PD, Lazar DA, Curra FP, et al. Ultrasound accelerates functional recovery after peripheral nerve damage. Neurosurgery 2001;48:1136-40; discussion 1140-1.

27. Nesser HJ, Karia DH, Tkalec W, et al. Therapeutic ultrasound in cardiology. Herz 2002;27:269-78.

28. Toth ZE, Leker RR, Shahar T, et al. The combination of granulocyte colony-stimulating factor and stem cell factor significantly increases the number of bone marrow-derived endothelial cells in brains of mice following cerebral ischemia. Blood 2008;111:5544-52.

29. Schlesinger M, Bendas G. Vascular cell adhesion molecule-1 (VCAM-1)--an increasing insight into its role in tumorigenicity and metastasis. Int $\mathrm{J}$ Cancer 2015;136:2504-14.

30. Ode Y, Aziz M, Wang P. CIRP increases ICAM-1+ phenotype of neutrophils exhibiting elevated iNOS and NETs in sepsis. J Leukoc Biol 2018;103:693-707.

31. Zhao LR, Singhal S, Duan WM, et al. Brain repair by hematopoietic growth factors in a rat model of stroke. Stroke 2007;38:2584-91.

32. Carbonneau CL, Despars G, Rojas-Sutterlin S, et al. Ionizing radiation-induced expression of INK4a/ARF in murine bone marrow-derived stromal cell populations interferes with bone marrow homeostasis. Blood 2012;119:717-26.

33. Leon CM, Barbosa CM, Justo GZ, et al. Requirement for PLC $\gamma 2$ in IL-3 and GM-CSF-stimulated MEK/ERK phosphorylation in murine and human hematopoietic stem/progenitor cells. J Cell Physiol 2011;226:1780-92.

34. Shi Y, Liu CH, Roberts AI, et al. Granulocyte-macrophage colony-stimulating factor (GM-CSF) and T-cell responses: what we do and don't know. Cell Res 2006;16:126-33.

35. Ofir R, Apte RN, Weinstein Y. Alterations in lymphokine secretion during leukemogenesis. Lymphokine Res 1988;7:217-26.

Cite this article as: Luo D, Chen W, Wang W, Chen J, Xu H, Chen J, Wang Y. Low-intensity pulsed ultrasound alleviating myelosuppression of Sprague-Dawley rats after combined treating by paclitaxel and carboplatin. Transl Cancer Res 2021;10(3):1183-1192. doi: 10.21037/tcr-20-3035 